V.2, N.1, 2018

DOI: $10.33947 / 2595-6264-v 2 n 1-3497$

\title{
AVALIAÇÃO DO PROCESSO DE REMEDIAÇÃO EM ÁREA CONTAMINADA POR HIDROCARBONETO
}

\section{EVALUATION OF PROCESS OF REMEDIATION IN AREA CONTAMINATED BY HYDROCARBON}

\author{
Pâmela Karen de Oliveira Simões da Silva'; Marisa Vianna Mesquita²
}

RESUMO: Com a formação dos grandes centros urbanos, grandes áreas industriais são criadas motivando interessantes incentivos econômicos, no entanto, tais crescimentos resultam em processos de degradação ambiental e impacto ambiental oriundo do uso excessivo dos recursos naturais e descarte irregular de resíduos. Diariamente, as indústrias de petróleo e derivados sofrem com problemas decorrentes de vazamentos, derrames e acidentes durante a exploração, refinamento, transporte, e operações de armazenamento. O presente trabalho tem como objetivo principal acompanhar uma metodologia de remediação de uma empresa "in situ" a fim de poder verificar e discutir a viabilidade do processo. No ano de 2007, a empresa através de uma investigação confirmatória de passivos ambientais, constatou por meio de poços de monitoramento, uma contaminação da água subterrânea (fase livre) por hidrocarboneto com espessura de aproximadamente $1 \mathrm{~cm}$ entre o evaporador e a casa da caldeira, bem como, fase dissolvida de Benzoapireno acima dos valores de intervenção para água subterrânea. Executou-se então, um detalhamento da pluma de fase livre e posteriormente a instalação de poços de monitoramento na área em questão. Mediante a análise da pluma de contaminante optou-se por um processo de remediação do tipo bombeamento "Pump and Treat" associado a caixas separadoras de água e óleo, onde a água retirada é direcionada ao tratamento e o óleo acondicionado para um devido descarte. A maior espessura de fase livre foi constatada no poço PB-01 no mês de abril de 2009 obtendo significativa diminuição em sua espessura já a partir dos meses seguintes juntamente com outros poços como PM-3 e PB-02. Com a execução contínua do processo e o constante monitoramento podese observar uma diminuição gradativa na espessura de fase livre nos anos de 2010 e a partir do mês de fevereiro de 2011 a inexistência do contaminante gerando o encerramento do bombeamento e comprovando a eficiência do processo, porém, com contínuo monitoramento da área ao longo de dois anos de acordo com as normas.

PALAVRAS-CHAVES: Degradação. Recuperação. Hidrocarbonetos.

ABSTRACT: With the formation of large urban centers, large industrial areas were created generating interesting economic incentives; however, they result in environmental degradation processes due to the excessive use of natural resources and environmental impacts in the irregular disposal of their waste. The oil industries suffer daily from problems arising from spills, spills and accidents during the exploration, refinement, transportation, and storage operations of oil and its derivatives. The present work has as main objective to evaluate the methodology used for remediation. In 2007, during the confirmatory investigation of Environmental Liabilities, by means of monitoring wells between the evaporator (EVP-01) and the boiler house, the contamination of groundwater was verified by the presence of hydrocarbon free phase with a thickness of approximately $1 \mathrm{~cm}$, and dissolved phase of Benzoapyrene above the intervention values for groundwater. A campaign was started to detail the free-stage plume found on the site and a further 7 monitoring wells were installed in the area in question. By means of the analysis of the contaminated plume, a "Pump and Treat" type recovery process was chosen, associated with water and oil separator boxes, where the water withdrawn is directed to the treatment and the oil is conditioned for proper disposal. The higher free-phase thickness was observed in well PB-01 in April 2009 with a value of $2.1 \mathrm{~cm}$, as of August 2009, a significant decrease in free-phase thickness was observed in wells PM-03, PB-01 and PB-02, gradually decreasing during the months of 2009 to 2010, as of February 2011 was no longer observed the presence of contamination by hydrocarbons in the form of free phase.

KEYWORDS: Contamination. Remediation. Hydrocarbons.

Discente do curso de Engenharia Ambiental - Universidade UNIVERITAS/UNG; pamelakaren-ambiental@outllok.com

Docente do curso de Engenharia Ambiental - Universidade UNIVERITAS/UNG; marisa.vianna@prof.ung.br 
AVALIAÇÃO DO PROCESSO DE REMEDIAÇÃO EM ÁREA CONTAMINADA POR HIDROCARBONETO Pâmela Karen de Oliveira Simões da Silva; Marisa Vianna Mesquita

\section{V.2, N.1, 2018}

\section{INTRODUÇÃO}

As contaminações ambientais provenientes de derrames de produtos derivados do petróleo juntamente com a falta de controle no armazenamento de produtos e ainda os vazamentos de tanques e tubulações subterrâneas contem objeto de grande preocupação para a sociedade e o poder público. A instalação inadequada de resíduos, o derrame de produtos durante seu manuseio, transporte e armazenamento são alguns dos exemplos que podem ser determinados como responsáveis por inúmeros casos de contaminação de solos e águas subterrâneas.

Segundo Silva (2005), os registros de vazamentos em tanques subterrâneos de petróleo e derivados têm sido episódios comuns constituindo uma importante fonte de poluição ambiental, modificando a qualidade ambiental. O número de ocorrências de acidentes envolvendo vazamentos de combustíveis em tanques de armazenamento tem colocado a população de áreas urbanas densamente povoadas a uma série de substancias tóxica à saúde humana, bem como, para o meio ambiente.

Os acidentes mais comuns estão relacionados aos hidrocarbonetos aromáticos, destacando-se o benzeno, tolueno, etilbenzeno e xileno (BTEX), os hidrocarbonetos alifáticos, além dos hidrocarbonetos totais de petróleo (RESCHEK, 2012). De acordo com Silva (2002), os compostos BTEX, constituintes da gasolina, apresentam maior solubilidade e mobilidade e são os responsáveis pelos maiores problemas de contaminação da água subterrânea. Além dos impactos associados ao meio ambiente, eventos que envolvem contaminação por petróleo e derivados têm sido componentes de preocupação devido à possibilidade de incidirem em riscos de explosão, incêndio além dos impactos à saúde pública.

Os frequentes derramamentos de petróleo e seus derivados registrados em solos brasileiros vêm motivando o desenvolvimento de tecnologias cada vez mais precisas para a descontaminação dessas matrizes (BENTO et al., 2003). Algumas tecnologias de remediação para solo e água subterrânea encontram-se citadas a seguir:

Extração Multifásica (MPE): sistema de extração multifásica que combina as técnicas de bioventilação e remoção de massa a vácuo, possibilitando a extração da fase livre, fase vapor, fase dissolvida na matriz do solo e estimulando o processo de biodegradação natural na zona não saturada (NOBRE e NOBRE, 2003; FURTADO, 2005).

Air Sparging: tecnologia que opera com altas taxas de fluxo de ar, a fim de manter contato constante entre a água e o solo e propiciar maior aeração da água subterrânea. O grupo-alvo de contaminante do Air Sparging são os compostos orgânicos voláteis (COVs) e combustíveis derivados de petróleo (NOBRE e NOBRE, 2003; FURTADO, 2005).

Biorremediação: utiliza-se de técnicas naturais para promover a remediação de uma área impactada através de microrganismos (bactérias e fungos), que degradam as substâncias ou compostos perigosos aos seres humanos e transformam em substâncias com pouca ou nenhuma toxicidade (TECNOHIDRO, 2001).

Bombeamento e tratamento (Pump \& Treat): sistema de contenção hidráulica convencional é provido de bombas (elétrica ou pneumática), para captação da água subterrânea impactada com tratamento adequado para os compostos de interesse, além disso, pode ser utilizado como espécie de barreira de contenção (linha de poços de bombeamento conhecida como barreira hidráulica), que altera as condições hidrológicas do local e impede que a contaminação siga o fluxo subterrâneo natural (NOBRE e NOBRE, 2008).

Mediante este cenário, o presente trabalho teve como objetivo poder acompanhar na prática uma metodologia de remediação, bem como, adquirir amplo conteúdo bibliográfico através de artigos científicos, livros e websites, auxiliando na assimilação do conteúdo explorado e facilitando o entendimento das informações contidas em relatórios técnicos sobre remediação de áreas contaminadas por hidrocarbonetos.

\section{ÁREA DE ESTUDO}

A empresa escolhida encontra-se ativa na área desde o ano de 2005 e seu ramo de atividade é responsável pela reciclagem de óleo combustível. Localizada na sub-região leste da Região Metropolitana de São Paulo, opera atualmente em um terreno cuja área total é de $3.124,00 \mathrm{~m}^{2}$ e a área construída de $478,04 \mathrm{~m}^{2}$. Possui altitude de $792 \mathrm{~m}$ e encontra-se inserida em um distrito industrial aprovado e denominado no Plano Diretor como Zona de Uso Predominantemente Industrial (ZUPI 1). A geologia da área é formada por horizontes argilosos e argilo-arenosos, que apresentam uma continuidade lateral, formando um pacote homogêneo em toda a área do empreendimento, estando inserida na Bacia Hidrográfica do Alto Tietê, esta de grande importância para captação de recursos hídricos para o Estado de 
AVALIAÇÃO DO PROCESSO DE REMEDIAÇÃO EM ÁREA CONTAMINADA POR HIDROCARBONETO

Pâmela Karen de Oliveira Simões da Silva; Marisa Vianna Mesquita

\section{V.2, N.1, 2018}

São Paulo. Vale ressaltar que a atual empresa comprou a área já com o contaminante, adquirindo também o passivo ambiental e tendo que arcar com todas as despesas de remediação.

\section{DESENVOLVIMENTO METÓDOLOGICO}

Após a instalação e no decorrer dos anos de operação da atual empresa, através de análises pode-se detectar a contaminação da água subterrânea por fase livre de hidrocarboneto com uma espessura de aproximadamente $1 \mathrm{~cm}$ e de fase dissolvida de benzo(a)pireno encontrado acima dos valores de intervenção para água subterrânea citados pela CETESB, 2005 (Tabela 1).

Com o objetivo de delimitar a pluma de contaminação por fase livre de hidrocarbonetos diagnosticada em torno de dois poços (PM-3 e PB-1), e adequar o local para o início das instalações do sistema de remediação, foram realizadas novas sondagens exploratórias e um amplo processo investigatório em toda a área da empresa. O resultado foi à instalação de poços obedecendo às normas da ABNT NBR 15.495-1, com 2 metros de seção filtrante na zona saturada e 01 metro de seção filtrante na zona não saturada.

Após o detalhamento da pluma constatou-se que a contaminação não ultrapassava os limites do empreendimento e durante o ano de 2007 então foram criados os 10 poços de monitoramento no pátio do empreendimento. Dois destes poços foram utilizados para bombeamento na fase de remedição, e os demais para monitoramento (PM-1; PM-2; PM-4; PM-5; PM-6; PM-7; PM-8). A metodologia utilizada para a remediação foi o processo "Pump and Treat", que atuou no empreendimento por aproximadamente 2 anos e meio.

O princípio de funcionamento do sistema "Pump and Treat" instalado na área consistiu de duas bombas pneumáticas submersas dentro dos poços de monitoramento (PB-1 e PB-2), que foram preenchidas com água e material em fase livre sobrenadante por gravidade. $\mathrm{O}$ ar comprimido então é direcionado para as bombas pneumáticas submersas por uma tubulação exclusiva expulsando o material contido nas bombas para uma segunda tubulação que é direcionada a uma caixa separadora de água e óleo, onde a água retirada é direcionada ao tratamento enquanto o óleo acondicionado para o devido descarte. Durante o período de operação do sistema foram extraídos e tratados, aproximadamente 520 litros de material contaminante.
Tabela 1: Valores orientados para solo e a água subterrânea proposta para o estado de São Paulo.

Table 1: Soil and ground water values proposed for the state of São Paulo.

\begin{tabular}{|c|c|c|c|c|c|c|c|}
\hline \multirow{3}{*}{ Substancia } & \multirow{3}{*}{$\begin{array}{l}\text { CAS } \\
\mathrm{n}^{*}\end{array}$} & \multicolumn{5}{|c|}{ Solo (me/kg) } & \multirow{3}{*}{$\begin{array}{l}\text { Agua sub } \\
\text { (10:/4) } \\
\text { Intervenção }\end{array}$} \\
\hline & & \multirow[b]{2}{*}{$\begin{array}{l}\text { Referençia } \\
\text { (a) }\end{array}$} & \multirow[b]{2}{*}{ Prevenção } & \multicolumn{3}{|c|}{ Intervençăo } & \\
\hline & & & & $\begin{array}{l}\text { Agricolala } \\
\text { AP Max }\end{array}$ & Residencial & Industrial & \\
\hline \multicolumn{8}{|c|}{ Hidrocarbonetos aromáticos voláteis } \\
\hline Benzeno & 71-43-2 & & 0,03 & 0,05 & 0,08 & 0,15 & 5 \\
\hline $\begin{array}{l}\text { Estireno } \\
\text { Etibenzen }\end{array}$ & $100-42-5$ & $<10$ & 0,2 & 25 & $\begin{array}{ll}35 \\
35\end{array}$ & 80 & 20 \\
\hline $\begin{array}{l}\text { Etibenzen } \\
\text { Tolueno }\end{array}$ & $\begin{array}{l}100-414-4 \\
108-88-3\end{array}$ & $\leqslant 0$ & $\begin{array}{l}6,2 \\
0,14\end{array}$ & $\begin{array}{l}30 \\
25\end{array}$ & ${ }_{20}^{35}$ & 80 & 700 \\
\hline Xilenos & $\begin{array}{l}1330-20-7 \\
100-7\end{array}$ & $\leqslant 0$ & 0,13 & 25 & 30 & 70 & 500 \\
\hline \multicolumn{8}{|c|}{ Hidrocarbonetos policiclicos aromaticos (2) } \\
\hline $\begin{array}{l}\text { Acenaftileno } \\
\text { Antraceno }\end{array}$ & $\begin{array}{l}208-96-8 \\
120-12-7\end{array}$ & $\leqslant L D$ & & & 15 & 30 & 175 \\
\hline $\begin{array}{l}\text { Antraceno } \\
\text { Benzo(a)antraceno }\end{array}$ & 56-55-3 & $\begin{array}{c}<L D \\
<L D\end{array}$ & 0,025 & $\begin{array}{l}7,5 \\
10\end{array}$ & 25 & 50 & 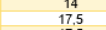 \\
\hline $\begin{array}{l}\text { Benzo(b)flfuoranteno } \\
\text { Benzzo(k)flforanteno }\end{array}$ & 205-99-2 $20 .-2$ & $\leqslant \leq D$ & 0.38 & $\begin{array}{c}3 \\
10\end{array}$ & $\begin{array}{l}30 \\
100\end{array}$ & $\begin{array}{c}70 \\
250\end{array}$ & $\begin{array}{l}17,5 \\
17,5\end{array}$ \\
\hline 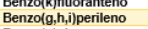 & $\begin{array}{l}200-1-26-9 \\
191-24.2\end{array}$ & $<\mathrm{LD}$ & 0,57 & 10 & 100 & 250 & $\begin{array}{l}17,5 \\
10,5\end{array}$ \\
\hline $\begin{array}{l}\text { Benzo(a)pireno } \\
\text { Criseno }\end{array}$ & 50-32-8 & $\leqslant L D$ & 0,052 & 0,4 & 1,5 & 4,0 & 0,7 \\
\hline $\begin{array}{l}\text { Criseno } \\
\text { Dibenzo(a,h)antraceno }\end{array}$ & 53-70-3-9 & $<\angle D$ & 8.1 & 0.15 & & & $\begin{array}{l}175 \\
175\end{array}$ \\
\hline Fenantreno & $85-01-8$ & $<\mathrm{LD}$ & 3,3 & 15 & 40 & 80 & \\
\hline Fluoranteno & $206-440$ & $\leqslant L D$ & 1,0 & 50 & 150 & 300 & 175 \\
\hline Indeno(1,2,3c,d)pireno & $193-39-5$ & $\angle L D$ & 0,031 & 2 & 30 & 80 & 17,5 \\
\hline Naftaleno & 193-39-5 & $<L D$ & 0,12 & 35 & 60 & 90 & 140 \\
\hline \multicolumn{8}{|l|}{ Benzen } \\
\hline Clorobenzeno(M & $108-90-7$ & $<L D$ & 0,41 & 40 & 45 & 110 & 140 \\
\hline $\begin{array}{l}\text { 1,2-Diclorobenze } \\
\text { 1,3-Diclorobenze }\end{array}$ & $\begin{array}{l}95-50-1 \\
541-7-1\end{array}$ & $<<D$ & . 0.73 & 150 & 200 & 400 & 1000 \\
\hline 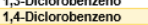 & $\begin{array}{l}106-46-7 \\
104-7\end{array}$ & $<L D$ & 0.39 & 50 & 70 & 150 & 300 \\
\hline $\begin{array}{l}\text { 1.2,3-Triclorobenzeno } \\
\text { 1,1,4-Triclorobenzenono }\end{array}$ & $\begin{array}{l}87-61-6 \\
120-2-1\end{array}$ & $\leqslant<D$ & $\begin{array}{l}0.01 \\
0.011\end{array}$ & $\begin{array}{l}5 \\
7 \\
7\end{array}$ & $\begin{array}{l}15 \\
20\end{array}$ & $\begin{array}{l}35 \\
40\end{array}$ & $\begin{array}{l}20(s) \\
20(s)\end{array}$ \\
\hline $\begin{array}{l}\text { 1,1,4-Inclorobenzeno } \\
\text { Hexaclorobenzeno }\end{array}$ & $118-74-1$ & $<L D$ & 0,003 & 0,005 & 0,02 & 0,05 & 0,56 \\
\hline
\end{tabular}

A operação toda do sistema de remediação foi automatizada e programada através de painel eletrônico de controle, que na utilização de um temporizador acionava a liberação de ar comprimido do compressor para os poços de bombeamento, iniciando assim o ciclo de bombeamento (Figura 1).

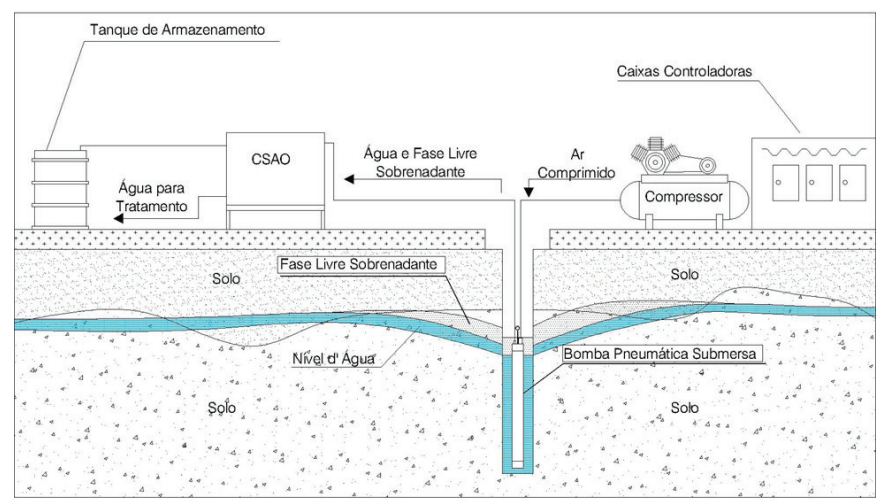

Figura 1: Esquema de funcionamento do sistema "Pump and Treat" instalado na área.

Figure 1: Diagram of operation of the "Pump and Treat" system installed in the área.

Fonte: PORFÍRIO (2011)

A coleta de dados do sistema de remediação foi captada quinzenalmente prevendo-se a realização dos seguintes itens: observação do devido funcionamento do sistema; realização dos reparos e ajustes necessários; medição do nível d’água (N.A); medição da espessura 
AVALIAÇÃO DO PROCESSO DE REMEDIAÇÃO EM ÁREA CONTAMINADA POR HIDROCARBONETO

Pâmela Karen de Oliveira Simões da Silva; Marisa Vianna Mesquita

V.2, N.1, 2018

de fase livre nos poços de monitoramento; ajuste da altura da bomba submersa para que esta estivesse sempre na região da fase livre; e a verificação da quantidade de contaminante removido dos poços de monitoramento.

\section{RESULTADOS E DISCUSSÃO}

Para um bom desempenho do processo de remediação tornou-se primordial o monitoramento do nível d'água (N.A.), este realizado através de um instrumento detector de diferentes comportamentos em meios fluidos acoplados a uma escala métrica com objetivo de determinar a profundidade do N.A. para o devido posicionamento das bombas submersas.

A Tabela 2 demonstra o acompanhamento do N.A. realizado durante os anos de 2009 a 2011 onde não há a observação de valores fora de um padrão hídrico, vale ressaltar que a pouca variação do nível d'água nos poços de monitoramento segue ciclos anuais coincidentes com a pluviometria média mensal da região, onde os níveis mais elevados corresponderam aos meses de maiores índices pluviométricos (dezembro a fevereiro) e os índices baixos aos meses de baixa pluviometria.

Tabela 02: Tabela da variação do N.A. (m), entre março de 2009 e agosto de 2011.

Table 02: Table of the variation of N.A. $(m)$, between March 2009 and August 2011.

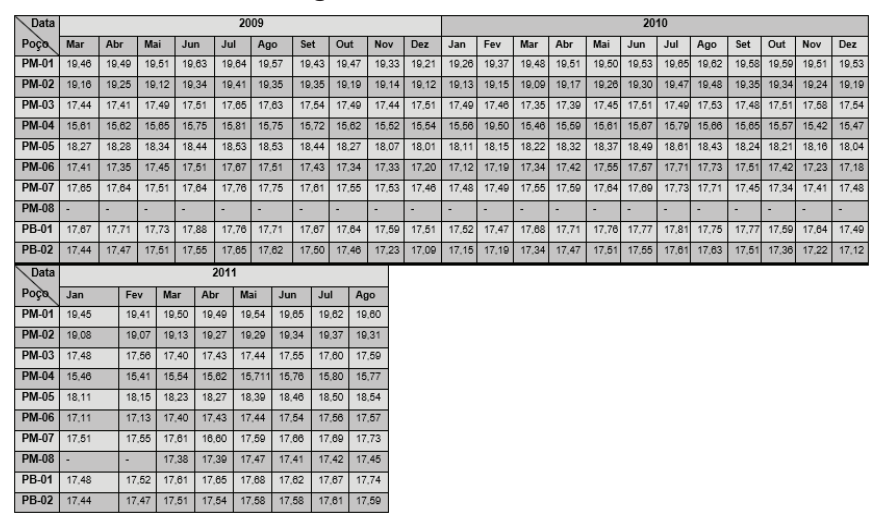

Fonte: PORFÍRIO (2011)

O monitoramento da espessura de fase livre nos poços de monitoramento é de grande importância para a viabilidade do procedimento utilizado na remediação da área contaminada, os dados obtidos direcionam a necessidade de ajustes quando necessários ou ao êxito final do processo.
A Tabela 3 demonstra os resultados das análises de monitoramento da espessura de fase livre no período de 2009 a 2011. Pode-se observar que o poço PB-01 no mês de abril/2009 obteve a maior espessura de fase livre de hidrocarbonetos com valor de 2,1 cm, apresentando uma significativa diminuição do contaminante conforme o processo de remediação fora instalado, a mesma diminuição de valor do contaminante pode ser observada nos demais poços também.

Tabela 03: Tabela da variação média de espessura da fase livre de hidrocarbonetos $(\mathrm{cm})$, entre março de 2009 e agosto de 2011.

Table 03: Table of the mean variation of the thickness of the hydrocarbon free phase $(\mathrm{cm})$, between March 2009 and August 2011.

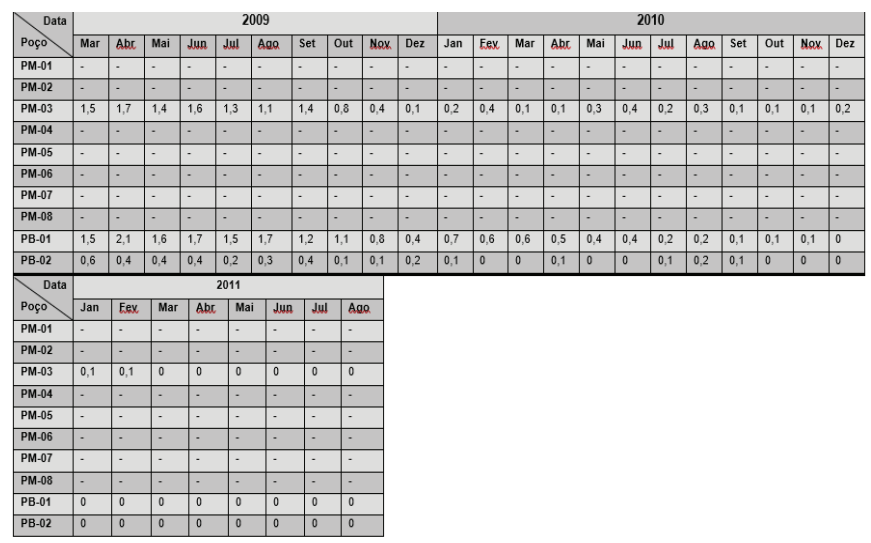

Fonte: PORFÍRIO (2011)

Assim como demonstrado na Tabela 3, o Gráfico 1 também denota a diminuição do contaminante a partir já da instalação do procedimento sendo visível a diminuição dos valores de espessura de fase livre já a partir do ano de 2010 e quase que zerando no ano de 2011, o que reforça a eficiência do processo utilizado. É importante salientar que posterior a todo o procedimento ainda pode-se observar uma oleosidade residual natural irrisória o que denota a continuidade do monitoramento da área.

Gráfico 1: Gráfico da variação média da Espessura de Fase Livre de hidrocarbonetos $(\mathrm{cm})$, entre março de 2009 e agosto de 2011.

Graphic 1: Graph of the average variation of the hydrocarbon free phase thickness (cm), between March 2009 and August 2011. 
AVALIAÇÃO DO PROCESSO DE REMEDIAÇÃO EM ÁREA CONTAMINADA POR HIDROCARBONETO

Pâmela Karen de Oliveira Simões da Silva; Marisa Vianna Mesquita

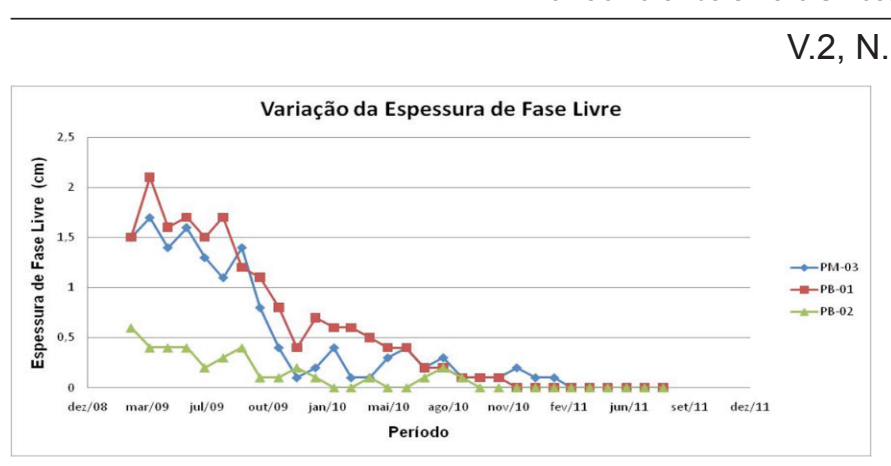

Fonte: PORFÍRIO (2011)

\section{CONCLUSÃO}

Dentre as metodologias de remediação, o bombeamento e tratamento realizado por "Pump and Treat" é atualmente um dos sistemas de tratamento de aquíferos mais utilizados no que consiste no bombeamento da água subsuperficial contaminada para tratamento em superfície facilitando assim a remoção dos contaminantes.
Assim como uma boa metodologia de remediação, pode-se verificar a necessidade de um bom estudo e levantamento de informações mediante o tipo de contaminante, sua distribuição em área e os efeitos de degradação.

De acordo com as características encontradas, o desenvolvimento de um bom planejamento e a escolha do processo resultou em uma redução significativa da presença de hidrocarbonetos em fase dissolvida, fato esse associado aos resultados positivos do sistema que se mostraram efetivos no tratamento da área.

O resultado da remediação atingiu as metas estipuladas, contemplando dessa forma a eficiência do sistema Pump and Treat, mesmo assim, após o encerramento da remediação foram ainda realizadas cinco campanhas de amostragem e análises de água subterrânea para monitoramento e controle da área. 


\section{REFERÊNCIAS}

ABNT- ASSOCIAÇÃO BRASILEIRA DE NORMAS TÉCNICAS. Norma para construção de Poços de Monitoramento de Águas Subterrâneas em Aquíferos Granulares (NBR 15.495-1). Rio de Janeiro, 2007. Disponível em: http://www.abnt.org.br. Acesso em: 11 set. 2017.

CETESB - COMPANHIA DE TECNOLOGIA DE SANEAMENTO AMBIENTAL. Decisão de Diretoria N ${ }^{\circ}$ 195-2005-E, de 23 de novembro de 2005. Dispõe sobre a aprovação dos Valores Orientadores para Solos e Águas Subterrâneas no Estado de São Paulo - 2005, em substituição aos Valores Orientadores de 2001 e dá outras providências. CETESB. São Paulo, 2005. Disponível em: http://www.cetesb.sp.gov.br. Acesso em: 25 set. 2017.

BENTO, F. M.; CAMARGO, F. A. O.; OKEKE, B.; FRANKENBERGER-JÚNIOR, W. T. Bioremediation of soil contaminated by diesel oil. In: Brazilian Congress of Microbiology, 2, Anais... Florianópolis/SC, 2003. (34) 65-68p.

FURTADO, M. Remediação de solo: para afastar o perigo que se esconde nos subterrâneos das grandes cidades, meios legais e econômicos devem fomentar obras de descontaminação. Revista Química e Derivados, São Paulo, maio. 2005, p. 28-44. Disponível em: http://www.quimica.com.br/revista/qd437/solos1.htm Acesso em: 14 jul. 2017.

NOBRE, M. M.; NOBRE, R. C. M. Remediação de solos: técnicas alternativas melhoram desempenho. 2003. Disponível em: http://www.quimicaederivados.htm Acesso em: 19 jul. 2017.
NOBRE, M. M.; NOBRE, R. C. M. Remediação de solos: técnicas alternativas melhoram desempenho. In Química e Derivados, v. 31. 2008. Disponível em: < http://www.quimica.com.br/revista/qd417/solo1.htm> Acesso em: 15. mar. 2017.

PORFÍRIO, L. Relatório de encerramento da atividades de remediação. Relatório Técnico. São Paulo, 2011.

RESCHKE, K. S. S. Estudos microbiológicos para tratamento de água subterrânea de áreas contaminadas por hidrocarbonetos. 2012. 100 p. Dissertação (Mestrado em Engenharia Civil) - Universidade do Vale do Rio dos Sinos/RS, 2012.

SILVA, M. A. B. Sistema de classificação Fuzzy para áreas contaminadas por petróleo e derivados. 2005. 221 p. Tese (Doutorado em Ciências da Engenharia) - Universidade Federal do Rio de Janeiro/RJ, 2005.

SILVA, R. L. B. Contaminação de poços rasos no bairro Brisamar, Itaguaí, RJ, por derramamento de gasolina: concentração de BTEX e avaliação da qualidade da água consumida pela população. 2002. 164 p. Tese (Doutorado em Ciências na área de Saúde Pública) - Escola Nacional de Saúde Pública da Fundação Oswaldo Cruz/RJ, 2002.

TECHOHIDRO. Tecnologias. São Paulo, 2001. Disponível em: http://www.tecnohidro.com.br/tec04.htm. Acesso em: 24 abr. 2017. 